\title{
CAREERS
}

TURNING POINT Social scientist hopes to engage the public in climate research p.227
EUROPEAN UNION Universities urge measures to improve researcher mobility p.227
NATUREJOBS For the latest career listings and advice www.naturejobs.com

\section{How to stay afloat}

\author{
When a lab runs out of money, there are a few strategies that it can use to get by.
}

\section{BY JEFFREY PERKEL}

$\mathrm{T}$ The world's economy is battered and bruised. More than three years after the start of the global recession, the European debt crisis threatens to derail a fragile financial recovery, and funding-agency budgets are suffering. Scientists are feeling the ill effects: many are having to make do with less, and there is a growing risk that researchers and laboratories will soon be caught between grants, unable to find new sources of funding when their current ones expire. "I'm not sure that I'm yet seeing the real impact of what's coming down the road," says John Kirby, dean of the College of the Environment and Life Sciences at the University of Rhode Island in Kingston, referring to the effects on his institution.

Across academia, scientists are planning for shortfalls, with mid-career researchers often the most at risk as funding agencies tailor grant programmes to younger researchers (see Nature 471, 399; 2011). "It is not clear that this development is recession-related, but its emphasis on 'stellar' individuals may have the effect of reducing significantly the funding options for many mid-career scientists," says Charles Dorman, chair of the department of microbiology at Trinity College Dublin.

But young researchers are far from immune: in some cases, university start-up packages are being stretched to allow for the increasing amount of time that a researcher needs to secure a first independent grant. Richard Schultz, associate dean for the natural sciences at the University of Pennsylvania's School of Arts and Sciences in Philadelphia, says that some of his institution's start-up packages, which typically last three years, may soon be recalculated to last for four. "There's a greater likelihood that it may take [new recruits] longer than we anticipated before they get their own extramural funding," he says.

There are steps that researchers can take to make up for deficits. Internal bridge funding can help in the short term, but it is best for scientists to plan ahead, broadening their search for support. Launching collaborations, varying funding sources and extending their work into unfamiliar areas could help anxious researchers to make their way out of the financial doldrums.

\section{CAUGHT BETWEEN GRANTS}

Despite their best efforts, researchers can sometimes find themselves without funding for months or even years. In such cases, bridge funding can often be arranged from within the researcher's home institution. Usually cobbled together from overheads and small amounts of money set aside in department and college budgets, such funding is generally limited in availability and quantity, and is awarded on a case-by-case basis. An institution's stopgap resources can vary widely, from a few thousand dollars per researcher to perhaps US $\$ 100,000$. Before deciding whether to approve bridge funding, a department chair or college dean will assess the researcher's publication and 
service record, funding history and likelihood of eventually winning an extramural grant.

Kirby recalls a case that came up when he was working as an associate dean for research at South Dakota State University in Brookings. A private entity funding a research project suddenly went bankrupt, leaving two graduate students bereft of

funds. The univer-

sity stepped in and moved the students into research-assistantship posts so that they could complete their work, he says. In another case, a successful faculty member requested about $\$ 50,000$ to finalize a data set and strengthen a grant application. Bridge funding allowed him to win the grant and continue his work.

But in tough eco-

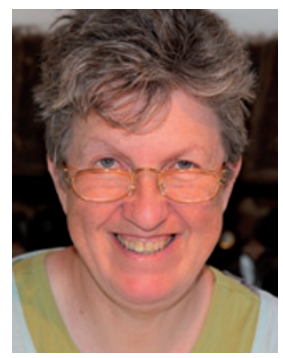

"The most important thing with a grant is to have a really interesting question." Mary Collins nomic times, such opportunities can be difficult to find. In cashstrapped Ireland, for instance, "the ability of universities to offer significant support to researchers facing a funding gap is very limited or even non-existent", says Dorman.

Carl Bauer, chair of the department of molecular and cellular biochemistry at Indiana University in Bloomington, helps to guide labs in his department out of financial straits. When a lab runs into trouble, the first thing Bauer does is to study its books carefully. Typically, such reviews don't entail micromanagement of reagent purchases or other equipment. Rather, the focus is on a lab's biggest expense: personnel. In the United States, graduate students can often be moved into teaching positions that require no funds from the principal investigator, but postdocs and technicians cannot. "Maybe I can keep them going for one more grant cycle, but after that point you have to let them go," says Bauer. Downsizing is almost inevitable at that point, says Mary Collins, dean of the faculty of life sciences at University College London. Bauer adds that he also examines a struggling scientist's past grant applications, gauging whether they have been competitive, because this could affect whether they will be able to gain an independent grant in the future.

\section{A DIVERSIFIED PORTFOLIO}

For those who have enough funding in the short term but worry about the medium and long term, experts recommend seeking money from multiple sources. A diverse portfolio of projects enables researchers to apply for a variety of grants. "It's like holding a basket of stocks in different areas," says Bauer. If one grant falls through, research priorities might take a hit, but the lab will survive on the others.

At any one time, Schultz aims to hold multiple grants with staggered start dates, to ensure that his lab's finances are relatively stable. He has several grants from the US National Institutes of Health coming up for renewal in the next few years; but given the current climate, he has "absolutely no confidence that any of them will be renewed. That, psychologically, is a bad place to be." Schultz plans to submit several further grant applications in partnership with colleagues - not his usual practice.

Researchers are increasingly establishing collaborations to hedge their bets. This can improve their chances of getting funding by, for instance, making a project more exciting through the use of innovative approaches and technologies, or more relevant to practical applications, says Collins. "You have to think a bit laterally," she adds. "The most important thing with a grant is to have a really interesting question. The second thing is to demonstrate that you have a clear means to providing some answers to that question. And if you have a technology that you really can't do or have never demonstrably done before, bringing in an expert who can do that as a collaborator is really helpful."

There is a caveat: weak collaborations could put applicants at a disadvantage. Adam Zlotnick, a biochemist at Indiana University, moved to his current post from the University of Oklahoma Health Science Center in Oklahoma City, where he had applied for a grant renewal in collaboration with an organic chemist who was hoping to synthesize a series of antiviral compounds. The application was unsuccessful, in part because the reviewers felt that the collaboration wasn't strong enough. Once at Indiana, Zlotnick found himself working just across the hall from another organic chemist. The two researchers developed a close interaction, talking face-to-face almost daily and sharing graduate students and postdocs. They won a grant on the basis of an application that emphasized teamwork and the interdisciplinary nature of their science. Zlotnick was fortunate: despite missing one grant cycle, he had another grant and start-up funds from Indiana to see him through. However, the start-up money had been intended to jump-start another project, which had to be slowed considerably as he addressed his funding gap. Although he has now regained independent funding, Zlotnick says his enthusiasm for new projects has been tempered.
Mere marriages of convenience are not enough to ensure funding success for collaborations, says Gareth Leng, head of the School of Biomedical Sciences at the University of Edinburgh, UK. "Grant panels see through them and they don't work out. So you've got to have a kind of a real marriage of love and enthusiasm," he says. "It's not a speed-dating thing." Leng adds that researchers should be careful not to diverge too far from their familiar research path when forming a collaboration or expanding the scope of their grant search. A radical change of direction can be detrimental in the current economic environment. "If you don't have the track record, you don't stand a chance," he notes.

\section{TUITION FEES}

Scientists who can not only sell their research, but can also find ways to use its content and methods to contribute to graduate-student training, may be able to tap into sources of funding related to higher education, notes Alan Tessier, an environmental-biology programme officer at the US National Science Foundation (NSF) in Arlington, Virginia. For example, a researcher could apply to lead a grant under a scheme such as the multimilliondollar NSF Integrated Graduate Education and Research Traineeship Program, or the NSF's Research Experience for Undergraduates initiative. "That includes support for graduate students in their lab," says Tessier.

There are other strategies. For instance, biologists working in vitro might attempt to move their research to an in vivo model to demonstrate its potential impact and importance for human health, strengthening their case with funders and expanding their technical reach. Scientists in other areas could try to incorporate new informatics or modelling tools into their work to expand their repertoires, show that their work is not stagnant and demonstrate the ability to adapt to new scientific questions, says Collins. In the end, stopgap funding solutions are most useful when they allow the lab to retain personnel. Lab groups can be fragile, notes Schultz. Personnel retain knowledge of the group's past projects and investigative track, so losing just one member can be very disruptive. Even if someone must temporarily work part-time while money is sought, the hope is that the lab will soon recover. "Then they can get moving," says Schultz, "and the lab won't go into what I call that death spiral." Bridge funding is a means of ensuring survival until long-term measures are possible. And whether an investigator has to rely on bridge funding or run a lab on a smaller budget, what ultimately matters, Schultz notes, is that the lab is running. "It's not that you're flourishing," he says. "Your purpose here is to survive."

Jeffrey Perkel is a freelance writer based in Pocatello, Idaho. 